follow that a deficient supply of oxygen to the respiratory centres has also something to do with asthma. In keeping with these remarks, it may be said that all circumstances productive of an excess of carbonic acid in the body and there fore of a deficiency of oxygen predispose to asthma. Cold and certain kinds of exercise do so pre-eminently, so does the ingestion of food, as happened in the case referred to above, where an attack of asthmatic cough came on pretty regularly two hours after dinner or at the time of the maximum production of carbonic acid from the meal.

A bad attack of asthma usually sets in with a sudden loud dry cough, coming on in the evening or at night; then follows a bronchial spasm during inspiration and the acute feeling of suffocation. After fighting for breath for ten twenty, and in some cases thirty hours the respiratory muscles become very tired, and also volition towards the respiratory effort. Moreover, the impediment to breathing prevents oxygen reaching the centres of respiration to promote the exercise of volition for forced breathing; hence the power of the will becomes less and less.

It is difficult to reconcile the fact that some asthmatic persons find themselves better under lower atmospheric pressures, while others, on the contrary, suffer the moment they leave the sea level. This is apparently due to the circumstance that some persons take in oxygen more readily under low pressures, and others under high pressures. I cannot help comparing asthma in those who suffer under low pressures to a sort of mountain sickness-the higher they go the worse they are, and the moment they return into the lower plains the asthma passes off as by magic. If mountain sickness can be prevented by training for the exercise of climbing under low pressure, why should not asthma also yield to the training of the respiration, carried out by practising the respiratory movements wanted to carry the tidal air through the lungs to oxidise the blood and emit the car bonic acid, while imparting to the circulation a better tendency to work in perfect harmony with the respiratory requirements?

Of all means of training the respiration I think cycling is the best. When a person first takes to cycling he is troubled with shortness of breath, his heart beats uncomfortably, and his legs get tired, but after some training these discomforts all disappear. Why should not people liable to attacks of asthma also train their respiration by such a kind of exercise, of course on condition of the heart and lungs being in perfect health ?

Cycling exercise first of all increases the depth of breathing, and that without fatigue, as the respiratory movements are automatic; at the same time it will accustom the rider instinctively to take in at each respiration the volume of air req iired to aerrate the blood and to eliminate a fixed proportiou of carbonic acid leaving in the circulation the precise amount compatible with health. In cycling, volition has also a good deal to do towards exercising the motor centres to take in the exact volume of oxygen required for that kind of work. Persons who do not care for cycling out of doors can take the same description of exercise at home by means of a dummy cycle.

My experience of the results of this treatment is unfortunately limited to one person; in which case it has proved eminently successful. This person took to bicycle riding three years ago for pleasure and afterwards on account of his health. He observed last summer that the attacks of asthma to which he was subject had become very much fewer, having only suffered two since last October, both of these being brought on by exposure to cold. The tightness and wheezing which occurred every night have nearly entirely disappeared, his sleep, which for years had been restless and broken, has very much improved, and all this he ascribes in a great measure to the use of the bicycle.

Allow me, gentlemen, to summarise shortly the Croonian lectures for the present year.

In the first lecture the oxygen of the atmosphere was shown to be the main agent of life, and Hermann's discovery that oxygen is an integral part of the tissues was appealed to with the object of explaining the remarkable tenacity of life of certain organisms-such as seeds. Next came an attempt to explain that it is not the oxygen of the air which, by offering itself to the tissues, produces the phenomena of metabolism, but that the living tissues call upon the oxygen to effect the changes they undergo. The influence of tissues as the prime movers in the matter was shown by the action of cold, which, through the nervous system, first increases their vitality and consequently their demand for oxygen, though when the cold is excessive it exerts an influence the reverse in its character. Muscular exercise invariably tends to the production of carbonic acid in excess; and this led to some remarks on the temperature of the body while in the act of ascending. It was concluded that with some people there is a slight increase and with others a slight fall of temperature.

The second lecture was prefaced with a short allusion to past investigations on human respiration; then followed the history of the different forms of breathing, which were illustrated by tracings (reduced in size) taken directly from the person under experiment. Attention was also drawn to the interesting phenomena of the absorption of oxygen in the body independently of the formation of carbonic acid, and a number of analyses were quoted showing to what extent this absorption takes place.

The third lecture was given up entirely to the influence of volition on the respiration. It was shown that volition towards any kind of muscular exercise, including forced breathing, is attended with the absorption of oxygen in excess, and that this oxygen is necessary towards the performance of volition, and, if necessary to volition it must be taken up by the motor centres of volition. A remarkable effect on the development of muscular force by this charging of the motor centres with oxygen was shown to take place. Finally, an experiment was described in which, volition becoming tired out, the muscles refused to work; this experiment being in keeping with Mosso's discovery.

The fourth lecture was devoted first of all to an account of the influence of climbing on the respiratory changes, and on the state of the respiratory phenomena at high altitudes. Next rebreathing and air breathed under pressure were considered, and it was explained that the effect of rebreathed air was apparently due more to the want of oxygen than the absorption of carbonic acid. After a few remarks on the effects of breathing mixtures of carbonic acid and common air, the lecture was concluded with an allusion to asthma, and a method of treatment suggested by the considerations which may be looked upon as the substance of these four lectures.

\section{CATS AND DIPHTHERIA.}

By WILLIAM WILLIAMS, M.A., M.D., D.P.H.OxoN., Medical Officer of Health to the Glamorgan County Council.

I have read with interest in the Britise Medical Jodranal of June 29th your remarks under the above heading, calling attention to the possible association of diphtheria in human beings and a suspicious illness amongst cats.

Some years ago, when acting as deputy and assistant to Dr. Thursfield, M.O.H., Shropshire, it was my lot to investigate a limited epidemic of diphtheria coincident with the deaths of three cats in one of the infected households.

In the month of December, 1889 , a limited epidemic of diphtheria took place at and in the neighbourhood of an agricultural village in Shropshire.

Thirteen cases, one of which proved fatal, occurred amongst five families. The first case occurred on November 7th, at the house of Mrs. L. (in the village), in the form of a slight sore throat, but that it was of an infectious nature is clear from the fact that the children at this house, eight in number, all suffered more or less severely since that date, including a grown.up daughter who came home at the time, and was laid up with a sore throat a few days after.

The second case-taken ill November 26th-was that of a child of Mr. B., whose house was quite a mile distant from the village.

The third case was that of a servant girl of Mrs. R., next door to Mrs. L. (in the village), who suffered very severely from marked diphtheria, but ultimately recovered. The fourth and fifth houses to be attacked were in the same treet.

At Mr. B.'s there were three cats kept. All these cats were 
taken ill and died. The symptoms observed were wasting, loss of appetite, inability to swallow, cough, and expectoration. They were ill some days before the children, and when ill were carried about and nursed by them. The children's illnesses were attributed to this cause by the household. The dates of the illnesses and deaths, as nearly as could be ascertained, were as follows :

First cat: taken ill November 18th and died December 1st

Second cat (yellow Persian): taken ill November 18th and died December 4th.

cat: taken ill November 25th and died December 7th.

Mr. B.'s two children were taken ill on November 26th and 27 th respectively, and the one taken ill on November 27 th died on December 9th, so that the illness and deaths of the cats preceded those of the children, and it appeared as if they had been connected. This was the opinion of the parents and people of the house. Mr. B.'s house was quite a mile from the village, and strict inquiry from the nurse and parents failed to elicit at first any connection. The nurse, however, was sister to Mrs. R., where the third case had occurred. She had visited her sister with Mr. B.'s children, but does not appear to have suffered from sore throat. Although at first sight the disease would appear to have originated with the cats, and the symptoms during life (above described) were such as would be anticipated if diphtheria affected the throats of the animals, this piece of evidence, showing a possible source of personal infection, renders it impossible to say whether the disease in the cats was any more than a coincidence. The first cat to die was examined by the medical attendant, who did not examine further than the throat, where he found no signs of inflammation, but a round worm of large size, which had choked the animal by blocking up the larynx.

Dr. Thursfield was of opinion that this was merely a symptomatic incident, and could be explained by the fact he had observed that during a febrile attack in human subjects the ascarides lumbricoides, doubtless rendered uncomfortable by the abnormal heat of the intestines, seek an exit by the bowel or the mouth. It is, moreover, possible that the violent efforts at coughing, obviously to clear the throat (described as a marked symptom in the cats) may have been chiefly, if not entirely, observed in this one cat. Another cat was unfortunately buried so as to be useless. The cat which died on December 7 th was secured, the symptoms of which were described as "coughing and something wrong in the throat." It was sent for examination to Dr. Klein, who reported that there was extensive disease of the lung not uncommon amongst cats.

Whether or not the illness of the children can be attributed to the cats or was merely coincident cannot be ascertained, but I consider that the incidents above related clearly show the desirability of examining the household cat or cats in every family infected with sore throat or diphtheria, and ever since it has been my custom to make careful inquiries after the cats.

The connection between infectious sore throat and diphtheria is well illustrated in the incidents of this epidemic, and I have often noticed that virulent diphtheria is very apt to occur shortly after the appearance of these ill-defined sore throats, and I am of the opinion that they are closely allied.

\section{NOTES OF A CASE OF SEROUS EFFUSION INTO THE PLEURA TREATED BY FREE INCISION AND DRAINAGE AFTER THE FAILURE OF REPEATED TAPPINGS.}

By RUTHERFORD MORISON, M.B., F.R.C.S., Senior Assistant Surgeon, Royal Infirmary, Newcastle-on-Tyne.

Dr. Samdel West's report, in the British Medical Journat of April 27th, 1895, of a case of serous effusion into the pleura treated successfully by free incision after the failure of repeated tapping, and the interest his paper excited when read at the Medical Society of London, induce me to send the notes of a case very similar to his. My case occurred in 1832 , and was recorded in a thesis presented by me to the University of Edinburgh, from which I have made the following extract.
E. A. L., aged 23, residing at West Hartlepool, was first seen on February 7th, 1882. The patient, a thin, pale, delicate-looking little woman, was unable to lie down because of difficulty in breathing. Her feet were swollen. The temperature was $100.6^{\circ} \mathrm{F}$.; the pulse was 96 , when she was lying quiet, but ran up to 120 when she was disturbed. In the abdomen a large apparently multilocular ovarian tumour was discovered; and the right chest was found to be dull all over on percussion. Breath sounds, vocal resonance, and vocal fremitus were absent.

The abdominal swelling had commenced with what, from her description, was an attack of peritonitis seven months earlier, and three months afterwards her abdomen was tapped by her medical attendant to relieve difficulty in breathing. A large but unknown quantity of clear fluid was removed by the tapping with some relief, but the abdomen rapidly refilled.

On February 9th, 1882, I tapped her abdomen and obtained 4 gallons of the glairy fluid commonly found in ovarian cysts. The tapping relieved her, but dyspncea at night was still troublesome.

On February 11th I aspirated the right pleura and withdrew the needle when 25 ounces of clear fluid had escaped. For two nights she was relieved; then the dyspnœa returned. On February 14th I aspirated again, slowly withdrawing through a small needle $4 \frac{1}{2}$ pints of fluid. The chest rapidly refilled, and by February 28th the dyspnœa had returned and the right chest appeared to be full again. I then decided to try if I could gain on the fluid by repeated small aspirations, and removed 1 pint of fluid. On March 2nd a second pint was aspirated; on March 6 th a third pint; and on March 10th a fourth pint. On. March 12th the chest seemed to be as full as ever, and the patient was steadily losing ground from fever, night sweats, the frequent tappings, and inability to eat.

On March 14th, 1882, with the antiseptic precautions practised at that time, under the carbolic spray, and without any anæsthetic, I made a free incision into the pleural cavity in the middle axillary line, and inserted 3 inches of large-bored india-rubber drainage tube. Fluid rushed from the tube during its escape. On several occasions, fearing that so sudden an emptying of the cavity might cause serious disturbance, I restrained the flow. The patient, however, only felt relief, and made no complaint of pain or discomfort, except a weak sensation. Large antiseptic dressings (carbolic gauze) were applied, but in spite of frequent change and liberal use of gauze the dressings were continually soaked during the first five days. For the first two days the tongue was dry and the temperature $100^{\circ}$ at night, otherwise there was no constitutional disturbance. On the sixth day after drainage the discharge suddenly ceased, and the dressing was for thefirst time found to be almost dry. From the sudden cessation of discharge I feared the tube might be blocked, but, on removing it. I found its lumen patent. A director passed into the pleural cavity also showed that it was empty. The percussion note at this time was tympanitic all over; there was a complete pneumothorax. The tube was introduced and retained for two days longer, when it was finally removed, as there was no discharge from it. The patient. steadily gained ground for a fortnight, at the end of which there were no physical signs of any chest derangement, except slightimpairment of the percussion note and some weakness of the breath sounds.

The abdominal swelling now began to be troublesome again, and a week later I performed ovariotomy. The operation was a difficult and severe one from dense adhesions to the parietes in the left lumbar region and the patient died half an hour after being put into bed. No post-mortem examination could be obtained.

REMARKs.-Before performing this operation I had seen Sir Joseph Lister open and drain knee-joints distended by fluid with success. This experience gave me confidence, and the result justified the means, for I have as little doubt as it is possible to have in the absence of a post-mortem examination that my patient's chest was well when she died. I have since always thought that an aseptic opening into the pleura was free from danger, that the proper surgical treatment of pleural effusions which resisted a moderate amount of tapping was to have them incised, drained, and dressed by a 